\title{
On a Thermoelastic Laminated Timoshenko Beam: Well Posedness and Stability
}

\author{
Baowei Feng (1) \\ Department of Economic Mathematics, Southwestern University of Finance and Economics, Chengdu 611130, China \\ Correspondence should be addressed to Baowei Feng; bwfeng@swufe.edu.cn
}

Received 7 January 2020; Accepted 3 February 2020; Published 28 February 2020

Guest Editor: Sundarapandian Vaidyanathan

Copyright (c) 2020 Baowei Feng. This is an open access article distributed under the Creative Commons Attribution License, which permits unrestricted use, distribution, and reproduction in any medium, provided the original work is properly cited.

In this paper, we are concerned with a linear thermoelastic laminated Timoshenko beam, where the heat conduction is given by Cattaneo's law. We firstly prove the global well posedness of the system. For stability results, we establish exponential and polynomial stabilities by introducing a stability number $\chi$.

\section{Introduction}

In this paper, we address the following thermoelastic laminated Timoshenko beam in $(0,1) \times(0, \infty)$ :

$$
\left\{\begin{array}{l}
\rho \omega_{t t}+G\left(\psi-\omega_{x}\right)_{x}+\delta \theta_{x}=0, \\
I_{\rho}(3 s-\psi)_{t t}-D(3 s-\psi)_{x x}-G\left(\psi-\omega_{x}\right)=0, \\
I_{\rho} s_{t t}-D s_{x x}+G\left(\psi-\omega_{x}\right)+\frac{4}{3} \gamma s+\frac{4}{3} \beta s_{t}=0, \\
\rho_{3} \theta_{t}+q_{x}+\delta \omega_{x t}=0 \\
\tau q_{t}+\alpha q+\theta_{x}=0,
\end{array}\right.
$$

which subject to the following boundary conditions:

$$
\begin{cases}\omega_{x}(0, t)=\psi(0, t)=s(0, t)=\theta(0, t)=0, & t \in(0, \infty), \\ \omega_{x}(1, t)=\psi(1, t)=s(1, t)=\theta(1, t)=0, & t \in(0, \infty),\end{cases}
$$

and initial conditions

$$
\left\{\begin{array}{c}
\omega(x, 0)=\omega_{0}(x), \psi(x, 0)=\psi_{0}(x), s(x, 0)=s_{0}(x), \theta(x, 0)=\theta_{0}(x), \quad x \in(0,1), \\
q(x, 0)=q_{0}(x), \omega_{t}(x, 0)=\omega_{1}(x), \psi_{t}(x, 0)=\psi_{1}(x), s_{t}(x, 0)=s_{1}(x), x \in(0,1)
\end{array}\right.
$$

where $\rho, G, I_{\rho}, D, \gamma, \beta, \rho_{3}, \delta, \tau$, and $\alpha$ are positive constants. $\theta(x, t)$ represents the difference temperature and $q(x, t)$ is the heat flux.
Laminated beam, which is a relevant research subject due to the high applicability of such materials in the industry, was firstly introduced by Hansen and Spies, see, for instance 
$[1,2]$. They introduced a mathematical model for two-layered beams with structural damping due to the interfacial slip which is given by

$$
\left\{\begin{array}{l}
\rho \omega_{t t}+G\left(\psi-\omega_{x}\right)_{x}=0 \\
I_{\rho}\left(3 s_{t t}-\psi_{t t}\right)-G\left(\psi-\omega_{x}\right)-D\left(3 s_{x x}-\psi_{x x}\right)=0 \\
I_{\rho} s_{t t}+G\left(\psi-\omega_{x}\right)+\frac{4}{3} \gamma s+\frac{4}{3} \beta s_{t}-D s_{x x}=0
\end{array}\right.
$$

where the coefficients $\rho, G, I_{\rho}, D, \gamma$, and $\beta$ are positive constants and represent density, shear stiffness, mass moment of inertia, flexural rigidity, adhesive stiffness, and adhesive damping parameter, respectively. The function $\omega(x, t)$ denotes the transversal displacement, $\psi(x, t)$ represents the rotational displacement, and $s(x, t)$ is proportional to the amount of slip along the interface at time $t$ and longitudinal spatial variable $x$. The third equation describes the dynamics of the slip.

Up till now, there are some results concerning laminated beam equations, which are mainly concerned with global existence and stability of the related system. By adding suitable damping effects, such as internal damping, (boundary) frictional damping, and viscoelastic damping, it was shown that if the linear damping terms are added in two of the three equations, system (4) is exponentially stable under the "equal wave speeds" assumption $\left(\rho / I_{\rho}\right)=(G / D)$. But if the damping terms are added in the three equations, then the system decays exponentially without the equal wave speeds assumption, see, for example, [3-17]. For thermoelastic laminated Timoshenko beam, there are few published works, we can mention the results due to Liu and Zhao [18] and Apalara [19]. In [18], the authors considered the following laminated beams with past history

$$
\left\{\begin{array}{l}
\rho \varphi_{t t}+G\left(\psi-\varphi_{x}\right)_{x}+\theta_{x}=0 \\
I_{\rho}(3 \omega-\psi)_{t t}-D(3 \omega-\psi)_{x x}+\int_{0}^{\infty} g(s)(3 \omega-\psi)_{x x}(t-s) \mathrm{d} s-G\left(\psi-\varphi_{x}\right)-\theta=0 \\
I_{\rho} \omega_{t t}-D \omega_{x x}+G\left(\psi-\varphi_{x}\right)+\frac{4}{3} \gamma \omega+\frac{4}{3} \beta \omega_{t}=0 \\
k \theta_{t}-\tau \theta_{x x}+\varphi_{x t}+(3 \omega-\psi)_{t}=0
\end{array}\right.
$$

together with the following boundary conditions:

$$
\begin{cases}\varphi_{x}(0, t)=\psi(0, t)=\omega(0, t)=\theta(0, t)=0, & t \in(0, \infty), \\ \varphi(1, t)=\psi_{x}(1, t)=\omega_{x}(1, t)=\theta_{x}(1, t)=0, & t \in(0, \infty) .\end{cases}
$$

They firstly proved the global well posedness of solutions to the system. The main results are the stability of the system. If $\beta \neq 0$, they proved the exponential and polynomial stabilities depending on the behavior of the kernel function $g$ only. If $\beta=0$, they established exponential stability in case of equal wave speeds assumption and lack of exponential stability in case of nonequal wave speeds assumption. Apalara [19] considered a laminated beam with second sound of the form

$$
\left\{\begin{array}{l}
\rho \omega_{t t}+G\left(\psi-\omega_{x}\right)_{x}=0 \\
I_{\rho}(3 s-\psi)_{t t}-D(3 s-\psi)_{x x}-G\left(\psi-\omega_{x}\right)+\delta \theta_{x}=0 \\
I_{\rho} s_{t t}-D s_{x x}+G\left(\psi-\omega_{x}\right)+\frac{4}{3} \gamma s+\frac{4}{3} \beta s_{t}=0 \\
\rho_{3} \theta_{t}+q_{x}+\delta(3 s-\psi)_{x t}=0 \\
\tau q_{t}+\alpha q+\theta_{x}=0
\end{array}\right.
$$

together with the following boundary conditions:

$$
\begin{cases}\omega_{x}(0, t)=\psi(0, t)=s(0, t)=q(0, t)=0, & t \in(0, \infty), \\ \omega(1, t)=\psi_{x}(1, t)=s_{x}(1, t)=\theta(1, t)=0, & t \in(0, \infty),\end{cases}
$$

and proved the global well posedness and established exponential and polynomial stabilities depending on the parameter

$$
\chi_{\tau}=\left(1-\frac{\tau \rho_{3} G}{\rho}\right)\left(\frac{D}{I_{\rho}}-\frac{G}{\rho}\right)-\frac{\tau G \delta^{2}}{\rho I_{\rho}} .
$$

One can also refer to two recent results of laminated beams with thermal damping in [20,21], and a result of a coupled hyperbolic equations with a heat equation of second sound in [22].

When $s=0$, system (4) reduces to the well-known Timoshenko system, which have been widely studied. There are so many papers on the Timoshenko system in the literature, most of those results recover the global well posedness, stability, and long-time dynamics by adding some kinds of damping. Here, we recall some works on the thermoelastic Timoshenko system. Muñoz Rivera and Racke [23] considered a Timoshenko system with thermoelastic dissipation and established exponential stability in case of equal wave speed assumption and polynomial stability if wave speeds are nonequal. Almeida Júnior et al. [24] studied 
a thermoelastic Timoshenko beam acting on shear force. They obtained the same stability results as in [23]. In addition, they proved that the polynomial decay is optimal. Fernández Sare and Racke [25] considered a Timoshenko system with second sound. They proved that the system is not exponentially stable even if the propagation speeds are equal. The results were generalized by Guesmia et al. [26]. Recently, Santos et al. [27] introduced a stability number $\chi_{r}$ for the system in [25] and established the exponential decay result for $\chi_{r}=0$ and polynomial decay for $\chi_{r} \neq 0$ by using the semigroup method. One can also find a stability result for the Timoshenko system with second sound in Apalara et al. [28]. Feng [29] considered a Timoshenko-Coleman-Gurtin system and studied the long-time dynamics of the system. We at last mention the contribution of Hamadouche and Messaoudi [30] and Aouadi and Boulehmi [31], where the authors considered two classes of nonuniform thermoelastic Timoshenko systems and proved global well posedness and established some stability results.

Our goals in the present work are to study the global well posedness and stability of systems (1)-(3). The main points are summarized as follows:

(i) We prove the global well posedness of systems (1)-(3) by using Lumer-Philips theorem. The main result is presented in Theorem 1.

(ii) We introduce a new stability number denoted by

$$
\chi=\tau \delta^{2} D-\left(D \rho-G I_{\rho}\right)\left(\frac{\tau \rho_{3} D}{I_{\rho}}-1\right)
$$

and we show that the system is exponential stable when $\chi=0$ and polynomial stable when $\chi \neq 0$. The main results are presented in Theorems 1 and 2 .

(iii) The proof of stability results is based on the multiplier method. Since the boundary conditions here we considered are different from those in Apalara [19], so the multipliers we will define are greatly different from the multipliers in Apalara [19].

It follows, from (1), that

$$
\begin{array}{r}
\frac{\mathrm{d}^{2}}{\mathrm{~d} t^{2}} \int_{0}^{1} \omega(x, t) \mathrm{d} x=0, \\
\tau \frac{\mathrm{d}}{\mathrm{d} t} \int_{0}^{1} q(x, t) \mathrm{d} x+\alpha \int_{0}^{1} q(x, t) \mathrm{d} x=0 .
\end{array}
$$

If we denote

$$
\begin{aligned}
& \bar{\omega}(x, t)=\omega(x, t)-\int_{0}^{1} \omega_{0}(x)-t \int_{0}^{1} \omega_{1}(x) \mathrm{d} x, \\
& \bar{q}(x, t)=q(x, t)-e^{-(\alpha / \tau) t} \int_{0}^{1} q_{0}(x) \mathrm{d} x,
\end{aligned}
$$

we easily verify that $(\bar{\omega}, \psi, s, \theta, \bar{q})$ satisfies (1) and in addition,

$$
\begin{aligned}
& \int_{0}^{1} \bar{\omega}(x, t) \mathrm{d} x=0, \\
& \int_{0}^{1} \bar{q}(x, t) \mathrm{d} x=0,
\end{aligned}
$$

$\forall t \geq 0$.

Hence, Poincaré's inequality holds for $\bar{\omega}$. In the following, we work with $\bar{\omega}$ and $\bar{q}$ but write $\omega$ and $q$ for convenience.

The remaining paper is planned as follows. In Section 2, we study the well posedness of the system. In Section 3, we establish the stability results. Throughout this paper, $c>0$ is a generic constant that changes from one inequality to another.

\section{Well Posedness}

We start by denoting the vector-valued function by $U$ :

$$
\begin{aligned}
& U=(\omega, \Phi, 3 s-\psi, 3 \Lambda-\Psi, s, \Lambda, \theta, q)^{T}, \\
& \text { with } \Phi=\omega_{t}, \\
& \Psi=\psi_{t}, \text { and } \Lambda=s_{t} .
\end{aligned}
$$

Then, systems (1)-(3) can be written as

$$
\left\{\begin{array}{l}
\frac{\mathrm{d}}{\mathrm{d} t} U(t)=\mathscr{A} U, \quad t>0, \\
U(0)=U_{0}=\left(\omega_{0}, \omega_{1}, 3 s_{0}-\psi_{0}, 3 s_{1}-\psi_{1}, s_{0}, s_{1}, \theta_{0}, q_{0}\right)^{T},
\end{array}\right.
$$

where the operator $\mathscr{A}$ is defined by

$$
\mathscr{A} U=\left(\begin{array}{c}
\Phi \\
-\frac{G}{\rho}\left(\psi-\omega_{x}\right)_{x}-\frac{\delta}{\rho} \theta_{x} \\
3 \Lambda-\Psi \\
\frac{D}{I_{\rho}}(3 s-\psi)_{x x}+\frac{G}{I_{\rho}}\left(\psi-\omega_{x}\right) \\
\Lambda \\
\frac{D}{I_{\rho}} s_{x x}-\frac{G}{I_{\rho}}\left(\psi-\omega_{x}\right)-\frac{4 \gamma}{3 I_{\rho}} s-\frac{4 \beta}{3 I_{\rho}} \Lambda \\
-\frac{1}{\rho_{3}} q_{x}-\frac{\delta}{\rho_{3}} \Phi_{x} \\
-\frac{\alpha}{\tau} q-\frac{1}{\tau} \theta_{x}
\end{array}\right) .
$$

We consider the following spaces: 


$$
\begin{aligned}
L_{*}^{2}(0,1) & =\left\{v \in L^{2}(0,1): \int_{0}^{1} v(x) \mathrm{d} x=0\right\}, \\
H_{*}^{1}(0,1) & =H^{1}(0,1) \cap L_{*}^{2}(0,1), \\
H_{*}^{2}(0,1) & =\left\{v \in H^{2}(0,1): v_{x}(0)=v_{x}(1)=0\right\} .
\end{aligned}
$$

$$
\begin{aligned}
\mathscr{H}= & H_{*}^{1}(0,1) \times L_{*}^{2}(0,1) \times H_{0}^{1}(0,1) \times L^{2}(0,1) \times H_{0}^{1}(0,1) \\
& \times L^{2}(0,1) \times L^{2}(0,1) \times L_{*}^{2}(0,1)
\end{aligned}
$$

be the Hilbert space equipped with the inner product

Let

$$
\begin{aligned}
(U, \widetilde{U})_{\mathscr{H}}= & \rho \int_{0}^{1} \Phi \widetilde{\Phi} \mathrm{d} x+I_{\rho} \int_{0}^{1}(3 \Lambda-\Psi)(3 \widetilde{\Lambda}-\widetilde{\Psi}) \mathrm{d} x+3 I_{\rho} \int_{0}^{1} \Lambda \widetilde{\Lambda} d x \\
& +\rho_{3} \int_{0}^{1} \theta \widetilde{\theta} \mathrm{d} x+\tau \int_{0}^{1} q \widetilde{q} \mathrm{~d} x+4 \gamma \int_{0}^{1} s \widetilde{s} \mathrm{~d} x+D \int_{0}^{1}(3 s-\psi)_{x}(3 \widetilde{\boldsymbol{s}}-\widetilde{\psi})_{x} \mathrm{~d} x \\
& +G \int_{0}^{1}\left(\psi-\omega_{x}\right)\left(\widetilde{\psi}-\widetilde{\omega}_{x}\right) \mathrm{d} x+3 D \int_{0}^{1} s_{x} \widetilde{s}_{x} \mathrm{~d} x
\end{aligned}
$$

The domain of $\mathscr{A}$ is given by

$$
D \mathscr{A}=\left\{\begin{array}{l|l}
U \in \mathscr{H} & \begin{array}{c}
\omega \in H_{*}^{2} 0,1 \cap H_{*}^{1} 0,1,3 s-\psi, s \in H^{2} 0,1 \cap H_{0}^{1} 0,1, \\
\Phi, q \in H_{*}^{1} 0,1,3 \Lambda-\Psi, \Lambda, \theta \in H_{0}^{1} 0,1
\end{array}
\end{array}\right\} .
$$

The well posedness result can be stated in the following theorem.

Theorem 1. Let $U_{0} \in \mathscr{H}$, then problems (1)-(3) admit a unique weak solution $U \in C\left(\mathbb{R}^{+}, \mathscr{H}\right)$. In addition, if $U_{0} \in D(\mathscr{A})$, then $U \in C\left(\mathbb{R}^{+}, D(\mathscr{A})\right) \cap C^{1}\left(\mathbb{R}^{+}, \mathscr{H}\right)$.

Proof. It is easy to obtain that, for any $U=(\omega, \Phi, 3 s-\psi$, $3 \Lambda-\Psi, s, \Lambda, \theta, q)^{T} \in D(\mathscr{A})$,

$$
(\mathscr{A} U, U)_{\mathscr{H}}=-4 \beta \int_{0}^{1} \Lambda^{2} \mathrm{~d} x-\alpha \int_{0}^{1} q^{2} \mathrm{~d} x \leq 0,
$$

which implies the operator $\mathscr{A}$ is a dissipative operator.

In what follows, we shall show the operator $I d-\mathscr{A}$ is surjective. In other words, given $F=\left(f_{1}, f_{2}, f_{3}, f_{4}\right.$, $\left.f_{5}, f_{6}, f_{7}, f_{8}\right) \in \mathscr{H}$, we will seek a solution $V=\left(v_{1}, v_{2}, v_{3}\right.$, $\left.v_{4}, v_{5}, v_{6}, v_{7}, v_{8}\right) \in D(\mathscr{A})$ of

$$
(I d-\mathscr{A}) V=F .
$$

We rewrite (21) as

$$
\left\{\begin{array}{l}
v_{1}-v_{2}=f_{1}, \\
\rho v_{2}-G v_{1 x x}-G v_{3 x}+3 G v_{5 x}+\delta v_{7 x}=\rho f_{2}, \\
v_{3}-v_{4}=f_{3}, \\
I_{\rho} v_{4}-D v_{3 x x}-3 G v_{5}+G v_{3}+G v_{1 x}=I_{\rho} f_{4}, \\
v_{5}-v_{6}=f_{5}, \\
\left(I_{\rho}+\frac{4}{3} \beta\right) v_{6}-D v_{5 x x}-G v_{3}-G v_{1 x}+\left(3 G+\frac{4}{3} \gamma\right) v_{5}=I_{\rho} f_{6} \\
\rho_{3} v_{7}+v_{8 x}+\delta v_{2 x}=\rho_{3} f_{7} \\
(\tau+\alpha) v_{8}+v_{7 x}=\tau f_{8}
\end{array}\right.
$$


which implies that

$$
\begin{aligned}
& v_{2}=v_{1}-f_{1}, \\
& v_{4}=v_{3}-f_{3}, \\
& v_{6}=v_{5}-f_{5},
\end{aligned}
$$

$$
v_{7 x}=-(\tau+\alpha) v_{8}+\tau f_{8} .
$$

We infer from (27) that

$$
v_{7}=-(\tau+\alpha) \int_{0}^{x} v_{8}(y) \mathrm{d} y+\tau \int_{0}^{x} f_{8}(y) \mathrm{d} y .
$$

Replacing (24)-(26) and (28) in (23), we see that

$$
\left\{\begin{array}{l}
\rho v_{1}-G v_{1 x x}-G v_{3 x}+3 G v_{5 x}-\delta(\tau+\alpha) v_{8}=\rho\left(f_{1}+f_{2}\right)-\tau f_{8}, \\
I_{\rho} v_{3}-D v_{3 x x}-3 G v_{5}+G v_{3}+G v_{1 x}=I_{\rho}\left(f_{3}+f_{4}\right) \\
\left(I_{\rho}+3 G+\frac{4}{3} \beta+\frac{4}{3} \gamma\right) v_{5}-D v_{5 x x}-G v_{3}-G v_{1 x}=I_{\rho}\left(f_{5}+f_{6}\right)+\frac{4}{3} \beta f_{5}, \\
-\rho_{3}(\tau+\alpha) \int_{0}^{x} v_{8}(y) \mathrm{d} y+v_{8 x}+\delta v_{1 x}=\rho_{3} f_{7}-\rho_{3} \tau \int_{0}^{x} f_{8}(y) \mathrm{d} y+\delta f_{1 x} . \\
\left.\widetilde{v}_{1}, \widetilde{v}_{3}, \widetilde{v}_{5}, \text { and }(\tau+\alpha) \int_{0}^{x} \widetilde{v}_{8}(y) \mathrm{d} y, \quad \mathscr{B}_{(}\left(v_{1}, v_{3}, v_{5}, v_{8}\right),\left(\widetilde{v}_{1}, \widetilde{v}_{3}, \widetilde{v}_{5}, \widetilde{v}_{8}\right)\right)=\mathscr{L}\left(\widetilde{v}_{1}, \widetilde{v}_{3}, \widetilde{v}_{5}, \widetilde{v}_{8}\right),
\end{array}\right.
$$

We multiply (29) by $\widetilde{v}_{1}, \widetilde{v}_{3}, \widetilde{v}_{5}$, and $(\tau+\alpha) \int_{0}^{x} \widetilde{v}_{8}(y) \mathrm{d} y$, respectively, and integrate their sum over $(0,1)$ to get the following variational formulation: where the bilinear form $\mathscr{B}:\left[H_{*}^{1}(0,1) \times H_{0}^{1}(0,1) \times\right.$ $\left.H_{0}^{1}(0,1) \times L_{*}^{2}(0,1)\right]^{2} \longrightarrow \mathbb{R}$ is given by

$$
\begin{aligned}
\mathscr{B}\left(\left(v_{1}, v_{3}, v_{5}, v_{8}\right),\left(\widetilde{v}_{1}, \widetilde{v}_{3}, \widetilde{v}_{5}, \widetilde{v}_{8}\right)\right) \\
=G \int_{0}^{1}\left(-v_{1 x}-v_{3}+3 v_{5}\right)\left(-\widetilde{v}_{1 x}-\widetilde{v}_{3}+3 \widetilde{v}_{5}\right) \mathrm{d} x+\rho \int_{0}^{1} v_{1} \widetilde{v}_{1} \mathrm{~d} x+I_{\rho} \int_{0}^{1} v_{3} \widetilde{v}_{3} \mathrm{~d} x \\
\quad+\left(3 I_{\rho}+4 \beta+4 \gamma\right) \int_{0}^{1} v_{5} \widetilde{v}_{5} \mathrm{~d} x+(\tau+\alpha) \int_{0}^{1} v_{8} \widetilde{v}_{8} \mathrm{~d} x+D \int_{0}^{1} v_{3 x} \widetilde{v}_{3 x} \mathrm{~d} x \\
\quad+3 D \int_{0}^{1} v_{5 x} \widetilde{v}_{5 x} \mathrm{~d} x+\rho_{3}(\tau+\alpha)^{2} \int_{0}^{1}\left(\int_{0}^{x} v_{8}(y) \mathrm{d} y\right)\left(\int_{0}^{x} \widetilde{v}_{8}(y) \mathrm{d} y\right) \mathrm{d} x
\end{aligned}
$$

and the linear form $\mathscr{L}:\left[H_{*}^{1}(0,1) \times H_{0}^{1}(0,1) \times H_{0}^{1}(0,1) \times\right.$ $\left.L_{*}^{2}(0,1)\right] \longrightarrow \mathbb{R}$ is defined by

$$
\begin{aligned}
\mathscr{L}\left(\widetilde{v}_{1}, \widetilde{v}_{3}, \widetilde{v}_{5}, \widetilde{v}_{8}\right)= & \int_{0}^{1}\left(\rho f_{1}+\rho f_{2}-\tau \delta f_{8}\right) \widetilde{v}_{1} \mathrm{~d} x+I_{\rho} \int_{0}^{1}\left(f_{3}+f_{4}\right) \widetilde{v}_{3} \mathrm{~d} x \\
& +\int_{0}^{1}\left[\left(3 I_{\rho}+4 \beta\right) f_{5}+3 I_{\rho} f_{6}\right] \widetilde{v}_{5} \mathrm{~d} x \\
& +\delta(\tau+\alpha) \int_{0}^{1} f_{1} \widetilde{v}_{8} \mathrm{~d} x \\
& +\int_{0}^{1}\left[\rho_{3} \tau(\tau+\alpha) \int_{0}^{x} f_{8}(y) \mathrm{d} y-\rho_{3}(\tau+\alpha) f_{7}\right] \\
& \cdot\left(\int_{0}^{x} \widetilde{v}_{8}(y) \mathrm{d} y\right) \mathrm{d} x .
\end{aligned}
$$

We denote the Hilbert space $V$ by

$$
V=H_{*}^{1}(0,1) \times H_{0}^{1}(0,1) \times H_{0}^{1}(0,1) \times L_{*}^{2}(0,1),
$$

equipped with the norm

$$
\begin{aligned}
\left\|\left(v_{1}, v_{3}, v_{5}, v_{8}\right)\right\|_{V}^{2}= & \left\|-v_{1 x}-v_{3}+3 v_{5}\right\|_{2}^{2}+\left\|v_{1}\right\|_{2}^{2} \\
& +\left\|v_{8}\right\|_{2}^{2}+\left\|v_{3 x}\right\|_{2}^{2}+\left\|v_{5 x}\right\|_{2}^{2} .
\end{aligned}
$$

It is easy to get that $\mathscr{B}(\cdot, \cdot)$ and $\mathscr{L}(\cdot)$ are bounded. Moreover there exists a positive constant $m$ such that

$$
\begin{aligned}
\mathscr{B}\left(\left(v_{1}, v_{3}, v_{5}, v_{8}\right),\left(v_{1}, v_{3}, v_{5}, v_{8}\right)\right) \\
=G \int_{0}^{1}\left(-v_{1 x}-v_{3}+3 v_{5}\right)^{2} \mathrm{~d} x+\rho \int_{0}^{1} v_{1}^{2} \mathrm{~d} x+I_{\rho} \int_{0}^{1} v_{3}^{2} \mathrm{~d} x \\
\quad+\left(3 I_{\rho}+4 \beta+4 \gamma\right) \int_{0}^{1} v_{5}^{2} \mathrm{~d} x+(\tau+\alpha) \int_{0}^{1} v_{8}^{2} \mathrm{~d} x+D \int_{0}^{1} v_{3 x}^{2} \mathrm{~d} x \\
\quad+3 D \int_{0}^{1} v_{5 x}^{2} \mathrm{~d} x+\rho_{3}(\tau+\alpha)^{2} \int_{0}^{1}\left(\int_{0}^{x} v_{8}(y) \mathrm{d} y\right)^{2} \mathrm{~d} x \\
\geq G \int_{0}^{1}\left(-v_{1 x}-v_{3}+3 v_{5}\right)^{2} \mathrm{~d} x+\rho \int_{0}^{1} v_{1}^{2} \mathrm{~d} x \\
\quad+(\tau+\alpha) \int_{0}^{1} v_{8}^{2} \mathrm{~d} x+D \int_{0}^{1} v_{3 x}^{2} \mathrm{~d} x+3 D \int_{0}^{1} v_{5 x}^{2} \mathrm{~d} x \\
\geq m\left\|\left(v_{1}, v_{3}, v_{5}, v_{8}\right)\right\|_{V}^{2} .
\end{aligned}
$$


Thus, $\mathscr{B}$ is coercive on $V \times V$. Consequently, using Lax-Milgram theorem, we conclude that (30) has a unique solution:

$$
\begin{aligned}
v_{1} & \in H_{*}^{1}(0,1), \\
v_{3}, v_{5} & \in H_{0}^{1}(0,1), \\
v_{8} & \in L_{*}^{2}(0,1),
\end{aligned}
$$

Substituting $v_{1}, v_{3}, v_{5}$, and $v_{8}$ into (24)-(26) and (28), respectively, we have

$$
\begin{array}{r}
v_{2} \in H_{*}^{1}(0,1), \\
v_{4}, v_{6} \in H_{0}^{1}(0,1), \\
v_{7} \in H_{0}^{1}(0,1) .
\end{array}
$$

Let $\widetilde{v}_{1} \in H_{0}^{1}(0,1)$ and denote

$$
\widehat{\widetilde{v}}_{1}(x)=\widetilde{v}_{1}(x)-\int_{0}^{1} \widetilde{v}_{1}(s) \mathrm{d} s,
$$

which gives us $\widehat{\widetilde{v}}_{1} \in H_{*}^{1}(0,1)$. Now we replace $\left(\widetilde{v}_{1}, \widetilde{v}_{3}, \widetilde{v}_{5}, \widetilde{v}_{8}\right)$ by $\left(\widehat{\widetilde{v}}_{1}, 0,0,0\right)$ in $(30)$ to obtain

$$
\begin{aligned}
& G \int_{0}^{1}\left(-v_{1 x}-v_{3}+3 v_{5}\right)\left(-\widehat{\widetilde{v}}_{1 x}\right) \mathrm{d} x+\rho \int_{0}^{1} v_{1} \widehat{\widetilde{v}}_{1} d x \\
& \quad=\int_{0}^{1}\left(\rho f_{1}+\rho f_{2}-\tau \delta f_{8}\right) \widehat{\widetilde{v}}_{1} \mathrm{~d} x,
\end{aligned}
$$

i.e.,

$$
\begin{aligned}
& G \int_{0}^{1} v_{1 x x} \widehat{\widetilde{v}}_{1} \mathrm{~d} x=\rho \int_{0}^{1} v_{1} \widehat{\widetilde{v}}_{1} \mathrm{~d} x-G \int_{0}^{1} v_{3 x} \widehat{\widetilde{v}}_{1} \mathrm{~d} x+3 G \int_{0}^{1} v_{5 x} \widehat{\widetilde{\widetilde{v}}}_{1} \mathrm{~d} x \\
& -\int_{0}^{1}\left(\rho f_{1}+\rho f_{2}-\tau \delta f_{8}\right) \widehat{\widetilde{\hat{v}}}_{1} \mathrm{~d} x, \quad \forall \widetilde{v}_{1} \in H_{0}^{1}(0,1),
\end{aligned}
$$

which yields

$$
\begin{aligned}
G v_{1 x x}= & \rho v_{1}-G v_{3 x}+3 G v_{5 x} \\
& -\left(\rho f_{1}+\rho f_{2}-\tau \delta f_{8}\right) \in L^{2}(0,1) .
\end{aligned}
$$

Thus,

$$
v_{1} \in H^{2}(0,1) .
$$

Moreover, (39) also holds for any $\phi \in C^{1}([0,1])$. Then, by using integration by parts, we obtain

$$
\begin{aligned}
& G v_{1 x}(1) \phi(1)-G v_{1 x}(0) \phi(0)-G \int_{0}^{1} v_{1 x x} \phi \mathrm{d} x \\
& \quad+\rho \int_{0}^{1} v_{1} \phi \mathrm{d} x-G \int_{0}^{1} v_{3 x} \phi \mathrm{d} x \\
& \quad+3 G \int_{0}^{1} v_{5 x} \phi \mathrm{d} x-\int_{0}^{1}\left(\rho f_{1}+\rho f_{2}-\tau \delta f_{8}\right) \phi \mathrm{d} x=0 .
\end{aligned}
$$

Then, we get for any $\phi \in C^{1}([0,1])$,

$$
G v_{1 x}(1) \phi(1)-G v_{1 x}(0) \phi(0)=0 .
$$

From (28), we obtain

$$
v_{7}(0)=v_{7}(1)=0 .
$$

Since $\phi$ is arbitrary, we get that $v_{1 x}(0)=v_{1 x}(1)=0$. Hence, $v_{1} \in H_{*}^{2}(0,1)$. Using similar arguments as above, we can obtain

$$
\begin{aligned}
v_{3}, v_{5} & \in H^{2}(0,1) \cap H_{0}^{1}(0,1), \\
v_{7} & \in H_{0}^{1}(0,1), \\
v_{8} & \in H_{*}^{1}(0,1) .
\end{aligned}
$$

Thus, $V=\left(v_{1}, v_{2}, v_{3}, v_{4}, v_{5}, v_{6}, v_{7}, v_{8}\right) \in D(\mathscr{A})$ and $\mathscr{A}$ is maximal. By using Lumer-Philips theorem, see, for example, Liu and Zheng [32] and Pazy [33], we end the proof of the theorem.

\section{Stability}

In this section, we study the stability of systems (1)-(3). More precisely, we establish exponential and polynomial decay results depending on $\chi$ defined by

$$
\chi=\tau \delta^{2} D-\left(D \rho-G I_{\rho}\right)\left(\frac{\tau \rho_{3} D}{I_{\rho}}-1\right) .
$$

The energy functional of systems (1)-(3) is defined by

$$
\begin{aligned}
E(t)= & E(\omega, \psi, s, \theta, q) \\
= & \frac{1}{2} \int_{0}^{1}\left[\rho \omega_{t}^{2}+I_{\rho}\left[(3 s-\psi)_{t}\right]^{2}+3 I_{\rho} s_{t}^{2}+\rho_{3} \theta^{2}\right. \\
& \left.+\tau q^{2}+4 \gamma s^{2}+D\left[(3 s-\psi)_{x}\right]^{2}+G\left(\psi-\omega_{x}\right)^{2}+3 D s_{x}^{2}\right] \mathrm{d} x .
\end{aligned}
$$

Now we give our stability results.

Theorem 2 (exponential decay). Suppose that $\chi=0$. For any initial data $U_{0} \in \mathscr{H}$, there exist two positive constants $\mu$ and $\eta$ such that the energy functional (48) satisfies

$$
E(t) \leq \mu e^{-\eta t}, \quad \forall t \geq 0 .
$$

Theorem 3 (polynomial decay). Suppose that $\chi \neq 0$. For any initial data $U_{0} \in D(\mathscr{A})$, there exists positive constant $\mu_{0}$ such that the energy functional (48) satisfies

$$
E(t) \leq \frac{\mu_{0}}{t}, \quad \forall t>0 .
$$

To prove Theorems 1 and 2, we need the following technical lemmas.

\subsection{Technical Lemmas}

Lemma 1. It holds that the energy functional $E(t)$ is nonincreasing and satisfies

$$
E^{\prime}(t)=-4 \beta \int_{0}^{1} s_{t}^{2} \mathrm{~d} x-\alpha \int_{0}^{1} q^{2} \mathrm{~d} x \leq 0 .
$$


Proof. Multiplying (1) by $\omega_{t},(3 s-\psi)_{t}, s_{t}$, $\theta$, and $q$, respectively, integrating the results by parts and using boundary condition (1), we easily get (51).

Lemma 2. Define the functional $F_{1}(t)$ by

$$
\begin{aligned}
F_{1}(t)= & I_{\rho} \int_{0}^{1}(3 s-\psi)_{t}(3 s-\psi) \mathrm{d} x \\
& -\rho \int_{0}^{1} \omega_{t} \int_{0}^{x}(3 s-\psi)(y) \mathrm{d} y \mathrm{~d} x .
\end{aligned}
$$

Then, we have for any $\varepsilon_{1}>0$,

$$
\begin{aligned}
F_{1}^{\prime}(t) \leq & -\frac{D}{2} \int_{0}^{1}\left[(3 s-\psi)_{x}\right]^{2} \mathrm{~d} x \\
& +\varepsilon_{1} \int_{0}^{1} \omega_{t}^{2} \mathrm{~d} x+c\left(1+\frac{1}{\varepsilon_{1}}\right) \int_{0}^{1}\left[(3 s-\psi)_{t}\right]^{2} \mathrm{~d} x \\
& +\frac{\delta^{2} c_{*}^{2}}{2 D} \int_{0}^{1} \theta^{2} \mathrm{~d} x
\end{aligned}
$$

where $c_{*}>0$ is the Poincare constant.

Proof. It follows from (1) that

$$
\begin{aligned}
F_{1}^{\prime}(t)= & D \int_{0}^{1}(3 s-\psi)_{x x}(3 s-\psi) \mathrm{d} x \\
& +G \int_{0}^{1}\left(\psi-\omega_{x}\right)(3 s-\psi) \mathrm{d} x+I_{\rho} \int_{0}^{1}\left[(3 s-\psi)_{t}\right]^{2} \mathrm{~d} x \\
& +G \int_{0}^{1}\left(\psi-\omega_{x}\right) \int_{0}^{x}(3 s-\psi)(y) \mathrm{d} y \mathrm{~d} x \\
& +\delta \int_{0}^{1} \theta_{x} \int_{0}^{x}(3 s-\psi)(y) \mathrm{d} y \mathrm{~d} x \\
& -\rho \int_{0}^{1} \omega_{t} \int_{0}^{x}(3 s-\psi)_{t}(y) \mathrm{d} y \mathrm{~d} x
\end{aligned}
$$

Using integration by parts and boundary condition (1), we arrive at

$$
\begin{aligned}
F_{1}^{\prime}(t)= & -D \int_{0}^{1}\left[(3 s-\psi)_{x}\right]^{2} \mathrm{~d} x+I_{\rho} \int_{0}^{1}\left[(3 s-\psi)_{t}\right]^{2} \mathrm{~d} x \\
& -\delta \int_{0}^{1} \theta(3 s-\psi) \mathrm{d} x \\
& -\rho \int_{0}^{1} \omega_{t} \int_{0}^{x}(3 s-\psi)_{t}(y) \mathrm{d} y \mathrm{~d} x .
\end{aligned}
$$

Then, by using Hölder's, Young's, and Poincaré's inequalities, we can get (53) from (57).

Lemma 3. The functional $F_{2}(t)$ defined by

$$
F_{2}(t)=\rho \int_{0}^{1}\left(\psi-\omega_{x}\right) \int_{0}^{x} \omega_{t}(y) \mathrm{d} y \mathrm{~d} x,
$$

satisfies for any $\varepsilon_{2}>0$,

$$
\begin{aligned}
F_{2}^{\prime}(t) \leq & -\frac{G}{2} \int_{0}^{1}\left(\psi-\omega_{x}\right)^{2} \mathrm{~d} x+\varepsilon_{2} \int_{0}^{1} \psi_{t}^{2} \mathrm{~d} x+c\left(1+\frac{1}{\varepsilon_{2}}\right) \int_{0}^{1} \omega_{t}^{2} \mathrm{~d} x \\
& +\frac{\delta^{2}}{2 G} \int_{0}^{1} \theta^{2} \mathrm{~d} x .
\end{aligned}
$$

Proof. Differentiating $F_{2}(t)$ with respect to $t$ and using (1), we see that

$$
\begin{aligned}
F_{2}^{\prime}(t)= & \rho \int_{0}^{1} \psi_{t} \int_{0}^{x} \omega_{t}(y) \mathrm{d} y \mathrm{~d} x-\rho \int_{0}^{1} \omega_{x t} \int_{0}^{x} \omega_{t}(y) \mathrm{d} y \mathrm{~d} x \\
& -G \int_{0}^{1}\left(\psi-\omega_{x}\right) \int_{0}^{x}\left(\psi-\omega_{y}\right)_{y} \mathrm{~d} y \mathrm{~d} x \\
& -\delta \int_{0}^{1}\left(\psi-\omega_{x}\right) \int_{0}^{x} \theta_{y} \mathrm{~d} y \mathrm{~d} x .
\end{aligned}
$$

Using integration by parts, we obtain

$$
\begin{aligned}
F_{2}^{\prime}(t)= & \rho \int_{0}^{1} \psi_{t} \int_{0}^{x} \omega_{t}(y) \mathrm{d} y \mathrm{~d} x+\rho \int_{0}^{1} \omega_{t}^{2} \mathrm{~d} x \\
& -G \int_{0}^{1}\left(\psi-\omega_{x}\right)^{2} \mathrm{~d} x-\delta \int_{0}^{1} \theta\left(\psi-\omega_{x}\right) \mathrm{d} x .
\end{aligned}
$$

Then, by using Young's inequality and Hölder's inequality, we can get (57).

Lemma 4. Define the functional $F_{3}(t)$ by

$$
F_{3}(t)=\tau \rho_{3} \int_{0}^{1} \theta \int_{0}^{x} q(y) \mathrm{d} y \mathrm{~d} x .
$$

Then, we can get for any $\varepsilon_{3}>0$,

$$
F_{3}^{\prime}(t) \leq-\frac{\rho_{3}}{2} \int_{0}^{1} \theta^{2} \mathrm{~d} x+\varepsilon_{3} \int_{0}^{1} \omega_{t}^{2} \mathrm{~d} x+c\left(1+\frac{1}{\varepsilon_{3}}\right) \int_{0}^{1} q^{2} \mathrm{~d} x .
$$

Proof. Differentiating $F_{3}$ with respect to $t$ and using (1), we obtain

$$
\begin{aligned}
F_{3}^{\prime}(t)= & -\tau \int_{0}^{1} q_{x} \int_{0}^{x} q(y) \mathrm{d} y \mathrm{~d} x-\tau \delta \int_{0}^{1} \omega_{x t} \int_{0}^{x} q(y) \mathrm{d} y \mathrm{~d} x \\
& -\rho_{3} \alpha \int_{0}^{1} \theta \int_{0}^{x} q \mathrm{~d} y \mathrm{~d} x-\rho_{3} \int_{0}^{1} \theta \int_{0}^{x} \theta_{y} \mathrm{~d} y \mathrm{~d} x .
\end{aligned}
$$

Integration by parts gives us

$$
\begin{aligned}
F_{3}^{\prime}(t)= & \tau \int_{0}^{1} q^{2} \mathrm{~d} y+\tau \delta \int_{0}^{1} \omega_{t} q \mathrm{~d} x-\rho_{3} \alpha \int_{0}^{1} \theta \int_{0}^{x} q \mathrm{~d} y \mathrm{~d} x \\
& -\rho_{3} \int_{0}^{1} \theta^{2} \mathrm{~d} x .
\end{aligned}
$$

By using Young's inequality and Hölder's inequality, we can get (61). 
Lemma 5. The functional $F_{4}(t)$ defined by

$$
F_{4}(t)=-\rho \rho_{3} \int_{0}^{1} \theta \int_{0}^{x} \omega_{t}(y) \mathrm{d} y \mathrm{~d} x,
$$

satisfies for any $\varepsilon_{4}>0$

$$
\begin{aligned}
F_{4}^{\prime}(t) \leq & -\frac{\rho \delta}{2} \int_{0}^{1} \omega_{t}^{2} \mathrm{~d} x+\varepsilon_{4} \int_{0}^{1}\left(\psi-\omega_{x}\right)^{2} \mathrm{~d} x+c\left(1+\frac{1}{\varepsilon_{4}}\right) \int_{0}^{1} \theta^{2} \mathrm{~d} x \\
& +\frac{\rho}{2 \delta} \int_{0}^{1} q^{2} \mathrm{~d} x .
\end{aligned}
$$

Proof. We take the derivative of $F_{4}$ and use (1) and integrate by parts to obtain

$$
\begin{aligned}
F_{4}^{\prime}(t)= & \rho \int_{0}^{1} q_{x} \int_{0}^{x} \omega_{t}(y) \mathrm{d} y \mathrm{~d} x+\rho \delta \int_{0}^{1} \omega_{x t} \int_{0}^{x} \omega_{t}(y) \mathrm{d} y \mathrm{~d} x \\
& +\rho_{3} G \int_{0}^{1} \theta \int_{0}^{x}\left(\psi-\omega_{y}\right)_{y}(y) \mathrm{d} y \mathrm{~d} x \\
& +\rho_{3} \delta \int_{0}^{1} \theta \int_{0}^{x} \theta_{y}(y) \\
= & -\rho \int_{0}^{1} q \omega_{t} \mathrm{~d} x-\rho \delta \int_{0}^{1} \omega_{t}^{2} \mathrm{~d} x \\
& +\rho_{3} G \int_{0}^{1} \theta\left(\psi-\omega_{x}\right) \mathrm{d} x+\rho_{3} \delta \int_{0}^{1} \theta^{2} \mathrm{~d} x .
\end{aligned}
$$

Lemma 6. Define the functional $F_{5}(t)$ by

$$
\begin{aligned}
F_{5}(t)= & \tau G \delta I_{\rho} \int_{0}^{1}(3 s-\psi)_{t}\left(\psi-\omega_{x}\right) \mathrm{d} x \\
& -\tau \delta D \rho \int_{0}^{1} \omega_{t}(3 s-\psi)_{x} \mathrm{~d} x \\
& +\tau \rho_{3}\left(D \rho-G I_{\rho}\right) \int_{0}^{1} \theta(3 s-\psi)_{t} \mathrm{~d} x \\
& -\tau\left(D \rho-G I_{\rho}\right) \int_{0}^{1} q(3 s-\psi)_{x} \mathrm{~d} x
\end{aligned}
$$

Then, we have for any $\varepsilon_{5}>0$,

$$
\begin{aligned}
F_{5}^{\prime}(t) \leq & -\frac{\tau G \delta I_{\rho}}{2} \int_{0}^{1}\left[(3 s-\psi)_{t}\right]^{2} \mathrm{~d} x+c_{1} \int_{0}^{1} s_{t}^{2} \mathrm{~d} x \\
& +c_{2} \int_{0}^{1} \theta^{2} \mathrm{~d} x+c_{3} \int_{0}^{1}\left(\psi-\omega_{x}\right)^{2} \mathrm{~d} x \\
& +\varepsilon_{5} \int_{0}^{1}\left[(3 s-\psi)_{x}\right]^{2} \mathrm{~d} x+C_{\varepsilon_{5}} \int_{0}^{1} q^{2} \mathrm{~d} x \\
& +\chi \int_{0}^{1} \theta_{x}(3 s-\psi)_{x} \mathrm{~d} x
\end{aligned}
$$

where $c_{i}(i=1,2,3)$ are positive constants.

Proof. By differentiating $F_{5}$ with respect to $t$, we have

Then, using Young's inequality, we can get (65).

$$
\begin{aligned}
F_{5}^{\prime}(t)= & \underbrace{\tau G \delta I_{\rho} \int_{0}^{1}(3 s-\psi)_{t t}\left(\psi-\omega_{x}\right) \mathrm{d} x}_{:=I_{1}}+\tau G \delta I_{\rho} \int_{0}^{1}(3 s-\psi)_{t}\left(\psi-\omega_{x}\right)_{t} \mathrm{~d} x \\
& \underbrace{-\tau \delta D \rho \int_{0}^{1} \omega_{t t}(3 s-\psi)_{x} \mathrm{~d} x}_{:=I_{2}}-\tau \delta D \rho \int_{0}^{1} \omega_{t}(3 s-\psi)_{x t} \mathrm{~d} x \\
& \underbrace{+\tau \rho_{3}\left(D \rho-G I_{\rho}\right) \int_{0}^{1} \theta_{t}(3 s-\psi)_{t} \mathrm{~d} x}_{:=I_{3}}+\underbrace{\tau \rho_{3}\left(D \rho-G I_{\rho}\right) \int_{0}^{1} \theta(3 s-\psi)_{t t} \mathrm{~d} x}_{:=I_{5}} \\
& \underbrace{-\tau\left(D \rho-G I_{\rho}\right) \int_{0}^{1} q_{t}(3 s-\psi)_{x} \mathrm{~d} x}_{:=I_{6}}-\underbrace{\tau\left(D \rho-G I_{\rho}\right) \int_{0}^{1} q(3 s-\psi)_{x t} \mathrm{~d} x} .
\end{aligned}
$$

Using equation (1) and integrating by parts, we see that

$$
\begin{aligned}
I_{1}= & -\tau G \delta D \int_{0}^{1}(3 s-\psi)_{x}\left(\psi-\omega_{x}\right)_{x} \mathrm{~d} x \\
& +\tau G^{2} \delta \int_{0}^{1}\left(\psi-\omega_{x}\right)^{2} \mathrm{~d} x, \\
I_{2}= & \tau \delta D G \int_{0}^{1}\left(\psi-\omega_{x}\right)_{x}(3 s-\psi)_{x} \mathrm{~d} x \\
& +\tau \delta^{2} D \int_{0}^{1} \theta_{x}(3 s-\psi)_{x} \mathrm{~d} x,
\end{aligned}
$$

$$
\begin{aligned}
I_{3}= & -\tau\left(D \rho-G I_{\rho}\right) \int_{0}^{1} q_{x}(3 s-\psi)_{t} \mathrm{~d} x \\
& -\tau \delta\left(D \rho-G I_{\rho}\right) \int_{0}^{1} \omega_{x t}(3 s-\psi)_{t} \mathrm{~d} x, \\
I_{4}= & -\frac{\tau \rho_{3} D}{I_{\rho}}\left(D \rho-G I_{\rho}\right) \int_{0}^{1} \theta_{x}(3 s-\psi)_{x} \mathrm{~d} x \\
& +\frac{\tau \rho_{3} G}{I_{\rho}}\left(D \rho-G I_{\rho}\right) \int_{0}^{1} \theta\left(\psi-\omega_{x}\right) \mathrm{d} x,
\end{aligned}
$$




$$
\begin{aligned}
I_{5}= & \alpha\left(D \rho-G I_{\rho}\right) \int_{0}^{1} q(3 s-\psi)_{x} \mathrm{~d} x \\
& +\left(D \rho-G I_{\rho}\right) \int_{0}^{1} \theta_{x}(3 s-\psi)_{x} \mathrm{~d} x \\
I_{6}= & \tau\left(D \rho-G I_{\rho}\right) \int_{0}^{1} q_{x}(3 s-\psi)_{t} \mathrm{~d} x
\end{aligned}
$$

Inserting (70)-(75) into (69), we can obtain

$$
\begin{aligned}
F_{5}^{\prime}(t)= & \tau G^{2} \delta \int_{0}^{1}\left(\psi-\omega_{x}\right)^{2} \mathrm{~d} x+\tau G \delta I_{\rho} \int_{0}^{1} \psi_{t}(3 s-\psi)_{t} \mathrm{~d} x \\
& +\frac{\tau \rho_{3} G}{I_{\rho}}\left(D \rho-G I_{\rho}\right) \int_{0}^{1} \theta\left(\psi-\omega_{x}\right) \mathrm{d} x \\
& +\alpha\left(D \rho-G I_{\rho}\right) \int_{0}^{1} q(3 s-\psi)_{x} \mathrm{~d} x \\
& +\chi \int_{0}^{1} \theta_{x}(3 s-\psi)_{x} \mathrm{~d} x .
\end{aligned}
$$

Recalling $\psi=(\psi-3 s)+3 s$ and using Young's inequality, we conclude that

$$
\begin{aligned}
& \tau G \delta I_{\rho} \int_{0}^{1} \psi_{t}(3 s-\psi)_{t} \mathrm{~d} x \\
& =-\tau G \delta I_{\rho} \int_{0}^{1}\left[(3 s-\psi)_{t}\right]^{2} \mathrm{~d} x+3 \tau G \delta I_{\rho} \int_{0}^{1} s_{t}(3 s-\psi)_{t} \mathrm{~d} x \\
& \leq-\frac{\tau G \delta I_{\rho}}{2} \int_{0}^{1}\left[(3 s-\psi)_{t}\right]^{2} \mathrm{~d} x+c_{1} \int_{0}^{1} s_{t}^{2} \mathrm{~d} x \\
& \frac{\tau \rho_{3} G}{I_{\rho}}\left(D \rho-G I_{\rho}\right) \int_{0}^{1} \theta\left(\psi-\omega_{x}\right) \mathrm{d} x \\
& \leq c_{2} \int_{0}^{1} \theta^{2} \mathrm{~d} x+c_{3} \int_{0}^{1}\left(\psi-\omega_{x}\right)^{2} \mathrm{~d} x
\end{aligned}
$$

and for any $\varepsilon_{5}>0$,

$$
\begin{aligned}
\alpha\left(D \rho-G I_{\rho}\right) \int_{0}^{1} q(3 s-\psi)_{x} \mathrm{~d} x \leq & \varepsilon_{5} \int_{0}^{1}\left[(3 s-\psi)_{x}\right]^{2} \mathrm{~d} x \\
& +C_{\varepsilon_{5}} \int_{0}^{1} q^{2} \mathrm{~d} x
\end{aligned}
$$

which, together with (76)-(78), gives us (68).

Lemma 7. The functional $F_{6}(t)$ defined by

$$
F_{6}(t)=3 I_{\rho} \int_{0}^{1} s_{t} s \mathrm{~d} x+2 \beta \int_{0}^{1} s^{2} \mathrm{~d} x
$$

satisfies

$$
\begin{aligned}
F_{6}^{\prime}(t) \leq & -3 \gamma \int_{0}^{1} s^{2} \mathrm{~d} x-3 D \int_{0}^{1} s_{x}^{2} \mathrm{~d} x \\
& +c_{4} \int_{0}^{1}\left(\psi-\omega_{x}\right)^{2} \mathrm{~d} x+3 I_{\rho} \int_{0}^{1} s_{t}^{2} \mathrm{~d} x,
\end{aligned}
$$

where $c_{4}$ is a positive constant.

Proof. follows from (1) that

$$
\begin{aligned}
F_{6}^{\prime}(t)= & -3 D \int_{0}^{1} s_{x}^{2} \mathrm{~d} x-3 G \int_{0}^{1} s\left(\psi-\omega_{x}\right) \mathrm{d} x \\
& -4 \gamma \int_{0}^{1} s^{2} \mathrm{~d} x+3 I_{\rho} \int_{0}^{1} s_{t}^{2} \mathrm{~d} x .
\end{aligned}
$$

Young's inequality gives us (82).

\subsection{Exponential Stability: Proof of Theorem 1}

Proof. We define the functional $\mathscr{L}(t)$ by

$$
\begin{aligned}
\mathscr{L}(t)= & N E(t)+F_{1}(t)+N_{2} F_{2}(t)+N_{3} F_{3}(t) \\
& +N_{4} F_{4}(t)+N_{5} F_{5}(t)+F_{6}(t),
\end{aligned}
$$

where $N$ and $N_{i}(i=2,3,4,5)$ are positive constants that will be chosen later.

Note that

$$
\begin{aligned}
\int_{0}^{1} \psi_{t}^{2} \mathrm{~d} x & =\int_{0}^{1}[(3 s-\psi)-3 s]_{t}^{2} \mathrm{~d} x \\
& \leq 2 \int_{0}^{1}\left[(3 s-\psi)_{t}\right]^{2} \mathrm{~d} x+18 \int_{0}^{1} s_{t}^{2} \mathrm{~d} x .
\end{aligned}
$$

Replacing (84) in (57) and then combining (51)-(53), (57)-(68), and (82), we obtain

$$
\begin{aligned}
\mathscr{L}^{\prime}(t) \leq & -\left(4 \beta N-c_{1} N_{5}-18 \varepsilon_{2} N_{2}-3 I_{\rho}\right) \int_{0}^{1} s_{t}^{2} \mathrm{~d} x \\
& -\left(\frac{D}{2}-\varepsilon_{5} N_{5}\right) \int_{0}^{1}\left[(3 s-\psi)_{x}\right]^{2} \mathrm{~d} x \\
& -\left[\alpha N-c N_{3}\left(1+\frac{1}{\varepsilon_{3}}\right)-\frac{\rho}{2 \delta} N_{4}-C_{\varepsilon_{5}} N_{5}\right] \int_{0}^{1} q^{2} \mathrm{~d} x \\
& -\left[\frac{\rho \delta}{2} N_{4}-\varepsilon_{1}-\varepsilon_{3} N_{3}-c N_{2}\left(1+\frac{1}{\varepsilon_{2}}\right)\right] \int_{0}^{1} \omega_{t}^{2} \mathrm{~d} x \\
& -\left[\frac{\tau G \delta I_{\rho}}{2} N_{5}-2 \varepsilon_{2} N_{2}-c\left(1+\frac{1}{\varepsilon_{1}}\right)\right] \int_{0}^{1}\left[(3 s-\psi)_{t}\right]^{2} \mathrm{~d} x \\
& -\left(\frac{G}{2} N_{2}-\varepsilon_{4} N_{4}-c_{3} N_{5}-c_{4}\right) \int_{0}^{1}\left(\psi-\omega_{x}\right)^{2} \mathrm{~d} x \\
& -\left[\frac{\rho_{3}}{2} N_{3}-\frac{\delta^{2} c_{*}^{2}}{2 D}-\frac{\delta^{2}}{2 G} N_{2}-c N_{4}\left(1+\frac{1}{\varepsilon_{4}}\right)-c_{2} N_{5}\right] \int_{0}^{1} \theta^{2} \mathrm{~d} x \\
& -3 D \int_{0}^{1} s_{x}^{2} \mathrm{~d} x-3 \gamma \int_{0}^{1} s^{2} \mathrm{~d} x .
\end{aligned}
$$


Taking

$$
\begin{aligned}
& \varepsilon_{1}=1, \\
& \varepsilon_{2}=\frac{\tau G \delta I_{\rho}}{8 N_{2}} N_{5}, \\
& \varepsilon_{3}=\frac{1}{N_{3}} \\
& \varepsilon_{4}=\frac{G}{4 N_{4}} N_{2}, \\
& \mathcal{E}_{5}=\frac{D}{4 N_{5}},
\end{aligned}
$$

we obtain

$$
\begin{aligned}
\mathscr{L}^{\prime}(t) \leq & -\left(4 \beta N-c_{1} N_{5}-\frac{9}{4} \tau G \delta I_{\rho} N_{5}-3 I_{\rho}\right) \int_{0}^{1} s_{t}^{2} \mathrm{~d} x \\
& -\frac{D}{4} \int_{0}^{1}\left[(3 s-\psi)_{x}\right]^{2} \mathrm{~d} x \\
& -\left[\alpha N-c N_{3}\left(1+N_{3}\right)-\frac{\rho}{2 \delta} N_{4}-C_{\varepsilon_{5}} N_{5}\right] \int_{0}^{1} q^{2} \mathrm{~d} x \\
& -\left[\frac{\rho \delta}{2} N_{4}-2-c N_{2}\left(1+\frac{8 N_{2}}{N_{5} \tau G \delta I_{\rho}}\right)\right] \int_{0}^{1} \omega_{t}^{2} \mathrm{~d} x \\
& -\left(\frac{\tau G \delta I_{\rho}}{4} N_{5}-2 c\right) \int_{0}^{1}\left[(3 s-\psi)_{t}\right]^{2} \mathrm{~d} x \\
& -\left(\frac{G}{4} N_{2}-c_{3} N_{5}-c_{4}\right) \int_{0}^{1}\left(\psi-\omega_{x}\right)^{2} \mathrm{~d} x \\
& -\left[\frac{\rho_{3}}{2} N_{3}-\frac{\delta^{2} c_{*}^{2}}{2 D}-\frac{\delta^{2}}{2 G} N_{2}\right. \\
& -c N_{4}\left(1+\frac{4 N_{4}}{G N_{0}}\right)-c_{2} s_{x}^{2} \mathrm{~d} x-3 \gamma \int_{0}^{1} s^{2} \mathrm{~d} x . \int_{0}^{1} \theta^{2} \mathrm{~d} x
\end{aligned}
$$
that

At this point, we first choose $N_{5}>0$ large enough such

$$
\frac{\tau G \delta I_{\rho}}{4} N_{5}-2 c>0 .
$$

For fixed $N_{5}$, we take $N_{2}>0$ so large that

$$
\frac{G}{4} N_{2}-c_{3} N_{5}-c_{4}>0 \text {. }
$$

Then, we pick $N_{4}>0$ large so that

$$
\frac{\rho \delta}{2} N_{4}-2-c N_{2}\left(1+\frac{8 N_{2}}{N_{5} \tau G \delta I_{\rho}}\right)>0 \text {. }
$$

And then we choose $N_{3}$ so large that

$$
\frac{\rho_{3}}{2} N_{3}-\frac{\delta^{2} c_{*}^{2}}{2 D}-\frac{\delta^{2}}{2 G} N_{2}-c N_{4}\left(1+\frac{4 N_{4}}{G N_{2}}\right)-c_{2} N_{5}>0
$$

At last, we take $N>0$ large enough so that the functional $\mathscr{L}(t)$ is equivalent to the energy functional $E(t)$, i.e., there exist two positive constants:

$$
\beta_{1} E(t) \leq \mathscr{L}(t) \leq \beta_{2} E(t)
$$

and further so that

$$
\begin{array}{r}
\alpha N-c N_{3}\left(1+N_{3}\right)-\frac{\rho}{2 \delta} N_{4}-C_{\varepsilon_{5}} N_{5}>0 . \\
4 \beta N-c_{1} N_{5}-\frac{9}{4} \tau G \delta I_{\rho} N_{5}-3 I_{\rho}>0,
\end{array}
$$

Recalling (48), we infer that there exists a positive constant $\beta_{3}$ such that, for any $t>0$,

$$
\mathscr{L}^{\prime}(t) \leq-\beta_{3} E(t),
$$

which, along with (92), implies

$$
\mathscr{L}^{\prime}(t) \leq-\frac{\beta_{3}}{\beta_{2}} \mathscr{L}(t) .
$$

Integrating (95) over $(0, t)$, we have, for any $t>0$,

$$
\mathscr{L}(t) \leq \mathscr{L}(0) e^{-\left(\beta_{3} / \beta_{2}\right)=t},
$$

which, using (95) again, gives us (49). The proof of Theorem 1 is done.

3.3. Polynomial Stability: Proof of Theorem 2. In this section, we consider the case $\chi \neq 0$ to prove Theorem 2 .

Differentiating system (1) with respect to time, we obtain the following system:

$$
\left\{\begin{array}{l}
\rho \omega_{t t t}+G\left(\psi-\omega_{x}\right)_{x t}+\delta \theta_{x t}=0, \\
I_{\rho}(3 s-\psi)_{t t t}-D(3 s-\psi)_{x x t}-G\left(\psi-\omega_{x}\right)_{t}=0, \\
I_{\rho} s_{t t t}-D s_{x x t}+G\left(\psi-\omega_{x}\right)_{t}+\frac{4}{3} \gamma s_{t}+\frac{4}{3} \beta s_{t t}=0, \\
\rho_{3} \theta_{t t}+q_{x t}+\delta \omega_{x t t}=0, \\
\tau q_{t t}+\alpha q_{t}+\theta_{x t}=0,
\end{array}\right.
$$

which subject to the following boundary conditions:

$$
\begin{cases}\omega_{x t}(0, t)=\psi_{t}(0, t)=s_{t}(0, t)=\theta_{t}(0, t)=0, & t \in(0, \infty), \\ \omega_{x t}(1, t)=\psi_{t}(1, t)=s_{t}(1, t)=\theta_{t}(1, t)=0, & t \in(0, \infty) .\end{cases}
$$

For any initial data $U_{0} \in D(\mathscr{A})$, system (97) is well posed. Next, we introduce second-order energy functional $\widetilde{E}(t)$ by 


$$
\begin{aligned}
\widetilde{E}(t)= & E\left(\omega_{t}, \psi_{t}, s_{t}, \theta_{t}, q_{t}\right) \\
= & \frac{1}{2} \int_{0}^{1}\left[\rho \omega_{t t}^{2}+I_{\rho}\left[(3 s-\psi)_{t t}\right]^{2}+3 I_{\rho} s_{t t}^{2}+\rho_{3} \theta_{t}^{2}+\tau q_{t}^{2}+4 \gamma s_{t}^{2}\right. \\
& \left.+D\left[(3 s-\psi)_{x t}\right]^{2}+G\left[\left(\psi-\omega_{x}\right)_{t}\right]^{2}+3 D s_{x t}^{2}\right] \mathrm{d} x .
\end{aligned}
$$

By using the same arguments as in Lemma 3, we can get the second-order energy $\widetilde{E}(t)$ defined by (99) is nonincreasing and satisfies

$$
\widetilde{E}^{\prime}(t)=-4 \beta \int_{0}^{1} s_{t t}^{2} \mathrm{~d} x-\alpha \int_{0}^{1} q_{t}^{2} \mathrm{~d} x \leq 0 .
$$

In Lemma 6, we have proved that, for any $\varepsilon_{5}>0$,

$$
\begin{aligned}
F_{5}^{\prime}(t) \leq & -\frac{\tau G \delta I_{\rho}}{2} \int_{0}^{1}\left[\left(3 s-\psi_{t}\right)^{2} \mathrm{~d} x\right]+c_{1} \int_{0}^{1} s_{t}^{2} \mathrm{~d} x \\
& +c_{2} \int_{0}^{1} \theta^{2} \mathrm{~d} x+c_{3} \int_{0}^{1}\left(\psi-\omega_{x}\right)^{2} \mathrm{~d} x \\
& +\varepsilon_{5} \int_{0}^{1}\left[(3 s-\psi)_{x}\right]^{2} \mathrm{~d} x+C_{\varepsilon_{5}} \int_{0}^{1} q^{2} \mathrm{~d} x \\
& +\chi \int_{0}^{1} \theta_{x}(3 s-\psi)_{x} \mathrm{~d} x .
\end{aligned}
$$

Thanks to (1) and Young's inequality, we derive that

$$
\int_{0}^{1} \theta_{x}^{2} \mathrm{~d} x \leq c \int_{0}^{1} q^{2} \mathrm{~d} x+c \int_{0}^{1} q_{t}^{2} \mathrm{~d} x .
$$

Then, for any $\varepsilon_{5}>0$,

$$
\begin{aligned}
\chi \int_{0}^{1} \theta_{x}(3 s-\psi)_{x} \mathrm{~d} x \leq & \varepsilon_{5} \int_{0}^{1}\left[(3 s-\psi)_{x}\right]^{2} \mathrm{~d} x+C_{\varepsilon_{5}} \int_{0}^{1} \theta_{x}^{2} \mathrm{~d} x \\
\leq & \varepsilon_{5} \int_{0}^{1}\left[(3 s-\psi)_{x}\right]^{2} \mathrm{~d} x+C_{\varepsilon_{5}} \int_{0}^{1} q^{2} \mathrm{~d} x \\
& +C_{\varepsilon 5} \int_{0}^{1} q_{t}^{2} \mathrm{~d} x .
\end{aligned}
$$

Therefore, the derivative of $F_{5}$ satisfies

$$
\begin{aligned}
F_{5}^{\prime}(t) \leq & -\frac{\tau G \delta I_{\rho}}{2} \int_{0}^{1}\left[(3 s-\psi)_{t}\right]^{2} \mathrm{~d} x+c_{1} \int_{0}^{1} s_{t}^{2} \mathrm{~d} x \\
& +c_{2} \int_{0}^{1} \theta^{2} \mathrm{~d} x+c_{3} \int_{0}^{1}\left(\psi-\omega_{x}\right)^{2} \mathrm{~d} x \\
& +2 \varepsilon_{5} \int_{0}^{1}\left[(3 s-\psi)_{x}\right]^{2} \mathrm{~d} x+C_{\varepsilon_{5}} \int_{0}^{1} q^{2} \mathrm{~d} x+C_{\varepsilon_{5}} \int_{0}^{1} q_{t}^{2} \mathrm{~d} x .
\end{aligned}
$$

Proof. We define the functional $\tilde{\mathscr{L}}(t)$ by

$$
\begin{aligned}
\widetilde{\mathscr{L}}(t)= & N(E(t)+\widetilde{E}(t))+F_{1}(t)+N_{2} F_{2}(t) \\
& +N_{3} F_{3}(t)+N_{4} F_{4}(t)+N_{5} F_{5}(t)+F_{6}(t) .
\end{aligned}
$$

It follows from (51)-(53), (57)-(65), and (100)-(104) that

$$
\begin{aligned}
\tilde{\mathscr{L}}^{\prime}(t) \leq & -\left(4 \beta N-c_{1} N_{5}-18 \varepsilon_{2} N_{2}-3 I_{\rho}\right) \int_{0}^{1} s_{t}^{2} \mathrm{~d} x-\left(\frac{D}{2}-2 \varepsilon_{5} N_{5}\right) \int_{0}^{1}\left[(3 s-\psi)_{x}\right]^{2} \mathrm{~d} x \\
& -\left[\alpha N-c N_{3}\left(1+\frac{1}{\varepsilon_{3}}\right)-\frac{\rho}{2 \delta} N_{4}-C_{\varepsilon_{5}} N_{5}\right] \int_{0}^{1} q^{2} \mathrm{~d} x \\
& -\left[\frac{\rho \delta}{2} N_{4}-\varepsilon_{1}-\varepsilon_{3} N_{3}-c N_{2}\left(1+\frac{1}{\varepsilon_{2}}\right)\right] \int_{0}^{1} \omega_{t}^{2} \mathrm{~d} x \\
& -\left[\frac{\tau G \delta I_{\rho}}{2} N_{5}-2 \varepsilon_{2} N_{2}-c\left(1+\frac{1}{\varepsilon_{1}}\right)\right] \int_{0}^{1}\left[(3 s-\psi)_{t}\right]^{2} \mathrm{~d} x \\
& -\left(\frac{G}{2} N_{2}-\varepsilon_{4} N_{4}-c_{3} N_{5}-c_{4}\right) \int_{0}^{1}\left(\psi-\omega_{x}\right)^{2} \mathrm{~d} x \\
& -\left[\frac{\rho_{3}}{2} N_{3}-\frac{\delta^{2} c_{*}^{2}}{2 D}-\frac{\delta^{2}}{2 G} N_{2}-c N_{4}\left(1+\frac{1}{\varepsilon_{4}}\right)-c_{2} N_{5}\right] \int_{0}^{1} \theta^{2} \mathrm{~d} x \\
& -3 D \int_{0}^{1} s_{x}^{2} \mathrm{~d} x-3 \gamma \int_{0}^{1} s^{2} \mathrm{~d} x-\left(\alpha N-C_{\varepsilon_{5}}\right) \int_{0}^{1} q_{t}^{2} \mathrm{~d} x
\end{aligned}
$$


With the same choice of constants as in Section 3.2, we further take $N>0$ so large that

$$
\alpha N-C_{\varepsilon_{5}}>0 .
$$

Noting that (48), we know that there exists a positive constant $\mu_{1}$ such that, for any $t>0$,

$$
\tilde{\mathscr{L}}^{\prime}(t) \leq-\mu_{1} E(t) \text {. }
$$

Since the energy functional $E(t)$ is positive and nonincreasing, we infer (108) that, for any $t>0$,

$$
t E(t) \leq \int_{0}^{t} E(s) \mathrm{d} s \leq \frac{1}{\mu_{1}}(\widetilde{\mathscr{L}}(0) t-n \tilde{\mathscr{L}} q(t)) \leq \frac{\tilde{\mathscr{L}}(0)}{\mu_{1}},
$$

which gives us

$$
E(t) \leq \frac{\mu_{0}}{t}, \quad \forall t>0 .
$$

Here, $\mu_{0}=\left(\widetilde{\mathscr{L}}(0) t / \mu_{1}\right)=\left(E(0)+\widetilde{E}(0) / \mu_{1}\right)$. The proof is complete.

Remark 1 . We point out that the functional $\tilde{\mathscr{L}}(t)$ is inequivalent to the energy functional $E(t)$. That is to say, (92) does not hold true.

\section{Data Availability}

No data were used during this study.

\section{Conflicts of Interest}

The author declares that there are no conflicts of interest regarding the publication of this paper.

\section{Acknowledgments}

This work was supported by the National Natural Science Foundation of China (Grant no. 11701465).

\section{References}

[1] S. W. Hansen, "A model for a two-layered plate with interfacial slip," in Control and Estimation of Distributed $\mathrm{Pa}$ rameter Systems: Nonlinear Phenomena, pp. 143-170, Birkhauser, Basel, Switzerland, 1994.

[2] S. W. Hansen and R. D. Spies, "Structural damping in laminated beams due to interfacial slip," Journal of Sound and Vibration, vol. 204, no. 2, pp. 183-202, 1997.

[3] M. S. Alves and R. N. Monteiro, "Exponential stability of laminated Timoshenko beams with boundary/internal controls," Journal of Mathematical Analysis and Applications, vol. 482, Article ID 123516, 2020.

[4] X.-G. Cao, D.-Y. Liu, and G.-Q. Xu, "Easy test for stability of laminated beams with structural damping and boundary feedback controls," Journal of Dynamical and Control Systems, vol. 13, no. 3, pp. 313-336, 2007.

[5] Z. Chen, W. Liu, and D. Chen, "General decay rates for a laminated beam with memory," Taiwanese Journal of Mathematics, vol. 23, no. 5, pp. 1227-1252, 2019.

[6] B. Feng, "Well-posedness and exponential decay for laminated Timoshenko beams with time delays and boundary feedbacks," Mathematical Methods in the Applied Sciences, vol. 41, no. 3, pp. 1162-1174, 2018.

[7] B. Feng, T. F. Ma, R. N. Monteiro, and C. A. Raposo, "Dynamics of laminated Timoshenko beams," Journal of Dynamics and Differential Equations, vol. 30, no. 4, pp. 1489-1507, 2018.

[8] G. Li, X. Kong, and W. Liu, "General decay for a laminated beam with structural damping and memory: the case of nonequal wave speeds," Journal of Integral Equations and Applications, vol. 30, no. 1, pp. 95-116, 2018.

[9] A. Lo and N.-e. Tatar, "Stabilization of a laminated beam with interfacial slip," Electronic Journal of Differential Equations, vol. 129, p. 14, 2015.

[10] A. Lo and N.-e. Tatar, "Uniform stability of a laminated beam with structural memory," Qualitative Theory of Dynamical Systems, vol. 15, no. 2, pp. 517-540, 2016.

[11] A. Lo and N.-e. Tatar, "Exponential stabilization of a structure with interfacial slip," Discrete and Continuous Dynamical Systems, vol. 36, no. 11, pp. 6285-6306, 2016.

[12] M. I. Mustafa, "Laminated Timoshenko beams with viscoelastic damping," Journal of Mathematical Analysis and Applications, vol. 466, no. 1, pp. 619-641, 2018.

[13] M. I. Mustafa, "On the stabilization of viscoelastic laminated beams with interfacial slip," Zeitschrift für Angewandte Mathematik und Physik, vol. 69, p. 33, 2018.

[14] M. I. Mustafa, "Boundary control of laminated beams with interfacial slip," Journal of Mathematical Physics, vol. 59, Article ID 051508, p. 9, 2018.

[15] C. A. Raposo, "Exponential stability for a structure with interfacial slip and frictional damping," Applied Mathematics Letters, vol. 53, pp. 85-91, 2016.

[16] C. A. Raposo, D. A. Z. Villanueva, S. D. M. Borjas, and D. C. Pereira, "Exponential stability for a structure with interfacial slip and memory," Poincare Journal of Analysis and Application, vol. 2, pp. 1-18, 2017.

[17] C. A. Raposo, O. V. Villagrán, J. E. Muñoz Rivera, and M. S. Alves, "Hybrid laminated Timoshenko beam," Journal of Mathematical Physics, vol. 58, Article ID 101512, 11 pages, 2017.

[18] W. Liu and W. Zhao, "Stabilization of a thermoelastic laminated beam with past history," Applied Mathematics \& Optimization, vol. 80, no. 1, pp. 103-133, 2019.

[19] T. A. Apalara, "Uniform stability of a laminated beam with structural damping and second sound," Zeitschrift für Angewandte Mathematik und Physik, vol. 68, p. 41, 2017.

[20] W. Liu, Y. Luan, Y. Liu, and G. Li, "Well-posedness and asymptotic stability to a laminated beam in thermoelasticity of type III," Mathematical Methods in the Applied Sciences, p. 19, 2019.

[21] L. Seghour, N. e. Tatar, and A. Berkani, "Stability of a thermoelastic laminated system subject to a neutral delay," Mathematical Methods in the Applied Sciences, vol. 43, no. 1, pp. 281-304, 2020.

[22] M. Afilal, S. Messaoudi, and A. Soufyane, "Stabilization of a coupled hyperbolic equations with a heat equation of second sound," Mediterranean Journal of Mathematics, vol. 14, no. 2, p. 39, 2017.

[23] J. E. Muñoz Rivera and R. Racke, "Mildly dissipative nonlinear Timoshenko systems-global existence and exponential stability," Journal of Mathematical Analysis and Applications, vol. 276, no. 1, pp. 248-278, 2002.

[24] D. d. S. Almeida Júnior, M. L. Santos, and J. E. Muñoz Rivera, "Stability to 1-D thermoelastic Timoshenko beam acting on 
shear force," Zeitschrift für angewandte Mathematik und Physik, vol. 65, no. 6, pp. 1233-1249, 2014.

[25] H. D. Fernández Sare and R. Racke, "On the stability of damped Timoshenko systems: Cattaneo versus Fourier law," Archive for Rational Mechanics and Analysis, vol. 194, no. 1, pp. 221-251, 2009.

[26] A. Guesmia, S. A. Messaoudi, and A. Soufyane, "Stabilization of a linear Timoshenko system with infinite history and applications to the Timoshenko-heat systems," Electronic Journal of Differential Equations, vol. 2012, no. 193, pp. 1-45, 2012.

[27] M. L. Santos, D. S. Almeida Júnior, and J. E. Muñoz Rivera, "The stability number of the Timoshenko system with second sound," Journal of Differential Equations, vol. 253, no. 9, pp. 2715-2733, 2012.

[28] T. A. Apalara, S. A. Messaoudi, and A. A. Keddi, "On the decay rates of Timoshenko system with second sound," Mathematical Methods in the Applied Sciences, vol. 39, no. 10, pp. 2671-2684, 2016.

[29] B. Feng, "On a semilinear Timoshenko-Coleman-Gurtin system: quasi-stability and attractors," Discrete \& Continuous Dynamical Systems-A, vol. 37, no. 9, pp. 4729-4751, 2017.

[30] T. Hamadouche and S. A. Messaoudi, "Existence and energy decay of a nonuniform Timoshenko system with second sound," Zeitschrift für Angewandte Mathematik und Physik, vol. 69, pp. 1-18, 2018.

[31] M. Aouadi and K. Boulehmi, "Asymptotic behavior of nonuniform Timoshenko beam acting on shear force with feedback controller," ZAMM-Journal of Applied Mathematics and Mechanics/Zeitschrift für Angewandte Mathematik und Mechanik, vol. 97, no. 12, pp. 1579-1599, 2017.

[32] Z. Liu and S. Zheng, Semigroups Associated with Dissipative Systems, Vol. 398, Chapman Hall/CRC, London, UK, 1999.

[33] A. Pazy, Semigroups of Linear Operators and Applications to Partial Differential Equations, Springer, New York, NY, USA, 1983. 